\title{
Recursos y obstáculos que afectan a los estudiantes de inglés en las escuelas secundarias norteamericanas ${ }^{1}$
}

\author{
María Fernández HawrylaK \\ Universidad de Burgos \\ Beth A. WASSELL \\ Universidad de Rowan \\ SARAh-Kate LaVAN \\ Departamento de Educación de Michigan
}

Recibido: 16 de octubre de 2012 / Aceptado: 6 de Octubre de 2013

ISSN: 1697-7467

\begin{abstract}
RESUMEN: Este artículo presenta un estudio cualitativo centrado en las experiencias de catorce estudiantes de inglés (ELL, English Language Learners) en escuelas secundarias urbanas norteamericanas cuya finalidad es conocer las estructuras que afectan a su capacidad de actuación, particularmente las relacionadas con la enseñanza y el aprendizaje. En primer lugar se ofrece una visión general de la literatura sobre las experiencias de los estudiantes de inglés en las escuelas de Estados Unidos. Posteriormente se describen las características y el diseño de la investigación, y los resultados obtenidos. Por último, se ofrecen conclusiones e implicaciones de la investigación. Las estructuras específicas de las aulas, de las escuelas y de la comunidad afectan a la capacidad del alumnado para acceder a los recursos necesarios para satisfacer sus necesidades sociales y de aprendizaje. Utilizando un marco metodológico de investigación narrativa encontramos que éstas incluían (i) Recursos: el espacio, el tiempo, y un esquema de atención que fueron creados por las prácticas docentes; (ii) Obstáculos: malas prácticas instructivas, falta de empatía hacia las experiencias de los estudiantes, y menores oportunidades de acceso al plan de estudios.
\end{abstract}

Palabras clave: Capacidad de actuación de los estudiantes; Inglés como segunda lengua; Aprendizaje de segundas lenguas; Escuela secundaria.

\section{Resources and Roadblocks that Affect English Language Learners in Urban High} Schools

\begin{abstract}
This article describes a qualitative study that focused on the experiences of 14 English Language Learner (ELL) students in urban high schools in the United States. The study investigated the structures that affected the students' agency, particularly related to teaching and learning. After describing relevant literature related the experiences of ELL students in the USA, the research design, findings, conclusions and implications are presented. Specific structures within classrooms, schools and the community impacted students' ability to access resources necessary to meet their academic and social needs and to learn effectively. Using a narrative inquiry framework, the findings revealed that resources included space, time, and a sense of caring created by teachers. The barriers included ineffective
\end{abstract}

${ }^{1}$ Esta investigación fue financiada mediante una beca interna no salarial de aptitud patrocinada por la Universidad de Rowan. 
instructional practices, a lack of empathy toward the students and their experiences, and fewer opportunities to access the curriculum.

Keywords: Agency; English as a Second Language; Second Language Learning; Secondary School

\section{INTRODUCCIÓN: PERSPECTIVAS DE LOS ESTUDIANTES ELL SOBRE SUS EXPE- RIENCIAS ESCOLARES}

Cada año el profesorado de todo el mundo se enfrenta a un número mayor de alumnos cultural y lingüísticamente diversos. En los Estados Unidos ha aumentado enormemente el número de estudiantes de inglés (ELL, English Language Learners), incrementándose el 53,2\% entre los cursos 1997-1998 y 2007-2008 cuando el número total de estudiantes sólo creció el 8,5\% en el mismo periodo (Batalova \& McHugh, 2010). Concretamente, el porcentaje de estudiantes ELL en las escuelas públicas norteamericanas era mayor en el curso 2009-2010 que en el 2000-2001 en todos los estados excepto en trece, y comparando únicamente los dos últimos cursos (08-09 y 09-10) había crecido en veintiocho estados (Aud et al., 2012). Ello significa que los estudiantes ELL poseen un dominio limitado del inglés, y que todos los docentes, y no sólo aquellos que enseñan inglés como segunda lengua (ESL, English as a Second Language), son responsables del aprendizaje de este alumnado ya que éstos representan menos del 1\% del profesorado en las escuelas públicas (Aud et al., 2012).

Fruto de esta situación han surgido nuevas investigaciones basadas en la práctica, muchas de ellas centradas en la aplicación de estrategias específicas de enseñanza para estos estudiantes. La literatura básica actual ofrece una gran cantidad de información acerca de cómo enseñar a los estudiantes de inglés; sin embargo, pocas fuentes proporcionan una visión sobre experiencias en las escuelas o de cómo éstas modelan el aprendizaje. Gran parte de la investigación sitúa el problema dentro del propio alumnado, mientras otra parte promueve visiones poco constructivas (Gutiérrez \& Orellana, 2006; Orellana \& Gutiérrez, 2006) en lugar de explorar las estructuras que apoyan o limitan el éxito académico. Las estructuras específicas en las aulas, en las escuelas y en la comunidad afectan a la capacidad de los estudiantes para acceder a los recursos apropiados para satisfacer sus necesidades educativas. Según los estudiantes, algunas han apoyado o limitado sus experiencias de aprendizaje durante el tiempo que han permanecido en la escuela secundaria.

Aunque para el profesorado es vital conocer la metodología apropiada para trabajar con estos estudiantes, convenimos con González \& Darling-Hammond en que los docentes también deben estar dispuestos a analizarse "a sí mismos, a la comunidad a la que sirven, a la comunidad de aprendizaje en la escuela, y a las relaciones de estas entidades entre $s i$ " (1997:131). Los educadores deben estarlo para conocer las vidas y experiencias de sus alumnos y para desarrollar empatía por sus situaciones. Para ello, los estudiantes necesitan oportunidades para reflexionar sobre los recursos que van encontrando para ser eficaces, potenciar y afrontar todos los aspectos de su educación.

Debido a que muchos estudiantes asisten a escuelas de bajo rendimiento en distritos urbanos que se enfrentan a retos como altos índices de pobreza, aumento de la rotación de profesores, bajas calificaciones en pruebas normalizadas y altas tasas de abandono escolar (Noguera, 2003), es imprescindible analizar tales estructuras haciendo hincapié en las palabras de los estudiantes y en sus puntos de vista. Así, la investigación realizada se centró en ofrecer a los estudiantes oportunidades para que pudieran proporcionar sus perspectivas sobre las propias experiencias escolares que permitiesen destacar aspectos importantes y poner de relieve su rol como aprendices.

Creemos que las percepciones de los estudiantes sobre sus experiencias desempeñan un papel importante en aquello que hacemos como educadores para proporcionar una instruc- 
ción apropiada y una orientación adecuada. En este sentido, nos centramos en dos temas clave que surgieron en nuestro análisis: los resultados académicos de los estudiantes, y los desafíos a los que se enfrentan en las escuelas (Wassell, Hawrylak \& LaVan, 2010). También describimos cómo la literatura tiende a reforzar un modelo de fracaso que rodea a los estudiantes ELL (Gutiérrez \& Orellana, 2006; Orellana, \& Gutiérrez, 2006).

Queremos poner de relieve los modos en que las escuelas y las aulas han sido o bien eficaces o bien insuficientes en el cumplimiento de las necesidades de estos estudiantes. Para ello intentamos encuadrar este artículo de manera que resalte la actuación de los alumnos utilizando sus opiniones, que nos pueden proporcionar información vital sobre las estructuras que han dado forma a su éxito en las escuelas, permitiéndonos comprender mejor la forma en que las escuelas y los maestros pueden servir más eficazmente a la población de estudiantes ELL.

\subsection{Enfoques desde la literatura}

Los investigadores han ido acumulado conocimientos sobre el desempeño académico de los estudiantes ELL y de sus experiencias en las escuelas a medida que su número aumentaba en las escuelas norteamericanas. Según Aud et al. (2012), el porcentaje de estudiantes ELL en las escuelas públicas norteamericanas era mayor en el curso 2009-2010 (algo más de un $10 \%$, aproximadamente 4,7 millones de estudiantes) que en el curso 2000-2001 (un $8 \%$, equivalente a 3,7 millones).

En muchos casos los investigadores se centran en los problemas o retos a los que se enfrentan en las aulas, en las escuelas o en las comunidades. En el corazón de estas dificultades se encuentra la afirmación de que los orígenes culturales y lingüísticos de los estudiantes ELL no son ventajosos para su éxito académico en las escuelas estadounidenses (Vang, 2005), lo que se manifiesta en su falta de dominio del idioma inglés, que por lo general precisa entre 4 y 7 años para alcanzar un nivel que les permita competir académicamente en igualdad de condiciones con sus compañeros angloparlantes (Hakuta, Butler \& Witt, 2000). Conjuntamente, a menudo tanto los estudiantes como sus padres carecen de los conocimientos necesarios para negociar con el sistema educativo de EE.UU. de manera exitosa (Louie, 2005; Gándara, 2010).

Por otra parte, profesores y estudiantes exacerban estos problemas en las escuelas. Muchos maestros comparten visiones de fracaso sobre la inclusión de los estudiantes ELL en sus aulas (Sacks \& Watnick, 2006). Al tiempo, estudiantes nativos de habla inglesa albergan ideologías hegemónicas y racistas sobre los estudiantes ELL, descritas por Serra i Salamé (2004) como la "retórica de la exclusión".

Portes \& Rumbaut (2001:251) sostienen que "el logro educativo es un proceso predecible y gobernado por las influencias contextuales de la familia y de la escuela, así como por los resultados de la adaptación anterior, incluyendo los tipos de aculturación y de ajuste de personalidad". Estos desafíos se pueden clasificar dentro de los límites borrosos de los factores sociales y de los basados en la escuela, cada uno de los cuales tiene fuertes implicaciones en las habilidades de los estudiantes ELL para tener éxito.

La literatura describe las razones principales que se hallan detrás de la falta de éxito académico de los estudiantes ELL, siendo importante explorar este hecho porque "lo bien que lo hagan y lo lejos que lleguen en la escuela regirá su posición final en el sistema 
social norteamericano" (Portes \& Rumbaut, 2001:234). Se ven obligados a hacer "un doble trabajo" ya que buscan al mismo tiempo el dominio de la lengua y del contenido académico (Short \& Fitzsimmons, 2007). Según los datos de la NAEP² de 2003, los estudiantes ELL de $4^{\circ}$ y $8^{\circ}$ iban dos o tres años a la zaga de sus compañeros de clase nativos de habla inglesa (Thompson, 2004, citado por Louie, 2005).

Estas diferencias pueden ser el resultado de la forma en la que son educados los estudiantes ELL, que Gándara, Rumberger, Maxwell-Jolly \& Callahan (2003) denuncian como significativamente inferior a la de los niños de habla inglesa. El estudio de estos autores se centró en las variables institucionales que influyen en el éxito de los estudiantes, concluyendo que los estudiantes ELL están expuestos a maestros menos competentes y a malas instalaciones, y suelen ser separados de sus compañeros de habla inglesa. El plan de estudios previsto para estos estudiantes es por lo general inferior, se cubre en un período menor de tiempo, e incorpora evaluaciones no válidas e ineficaces que no miden adecuadamente sus logros (Gándara et al., 2003). Además, el 70\% de los estudiantes ELL en los Estados Unidos se concentra en el $10 \%$ de las escuelas (conocidas como "High-LEP") principalmente en las zonas urbanas, lo que representa una población considerable de estudiantes ELL (Cosentino de Cohen, Deterding \& Chu Clewell, 2005). Según estos autores, los maestros de esas escuelas a menudo carecen de la licencia correspondiente.

Aunque la investigación descrita proporciona una amplia visión de los desafíos a los que se enfrentan los estudiantes ELL, los mensajes que se desprenden de los trabajos revisados deberían ser interpretados con precaución, ya que desafortunadamente la literatura sobre las experiencias de estos estudiantes en las escuelas transmite y refuerza un modelo de fracaso e impide identificar aspectos reparables (Orellana \& Gutiérrez, 2006).

\subsection{Las capacidades de los estudiantes ELL}

La actuación de los estudiantes, las formas en que acceden a los recursos apropiados que pueden utilizar en su beneficio y su interacción con las estructuras sociales e institucionales son importantes para alcanzar el éxito académico. Según Sewell (1992:16), las nociones de estructura y actuación existen dialécticamente. Las estructuras son "conjuntos de esquemas $y$ recursos mutuamente mantenedores que potencian y limitan el comportamiento social y que tienden a ser reproducidos". Estos recursos y esquemas culturales que conforman las estructuras están interrelacionados, ya que los recursos (que son reales) dan forma a los esquemas (que son virtuales) y viceversa.

La actuación se define como la energía de participante que se origina desde la conciencia de los esquemas o el control de los recursos. Se trata de "la capacidad del actor para reinterpretar y movilizar una variedad de recursos, en términos de esquemas culturales, distintos de los que en un principio constituían su colección de recursos" (Sewell, 1992:20).

En este estudio utilizamos la dialéctica entre estructura y actuación para: reflexionar sobre los esquemas y los recursos que intervienen en las experiencias de los estudiantes; examinar la forma en que los estudiantes ejercen su actuación mientras se encuentran en

\footnotetext{
${ }^{2}$ National Assessement of Education Progress.

${ }^{3}$ Limited English Proficient, dominio limitado del inglés.
} 
los contextos de las escuelas secundarias; y reconocer las estructuras institucionales que, según ellos, describen sus experiencias como estudiantes de secundaria. Nuestro interés se centró particularmente en los recursos y los esquemas que afectan al aprendizaje, al acceso al currículo y a las oportunidades para obtener dominio del inglés.

\section{DESCRIPCIÓN DE LA INVESTIGACIÓN}

\subsection{Tipo de investigación}

Fue diseñada en torno a la siguiente pregunta: ¿Qué ideas pueden compartir con nosotros los estudiantes ELL de las escuelas secundarias acerca de las estructuras que afectan a su actuación, en particular en lo que respecta a la enseñanza y el aprendizaje? (Wassell, Hawrylak \& LaVan, 2010).

Al explorar esta pregunta, se procuró ofrecer a los estudiantes la oportunidad de explicar al profesorado y a los formadores de docentes lo que ellos consideraban que era eficaz o ineficaz según su experiencia escolar.

Se eligió un diseño cualitativo informado por la indagación narrativa. El uso de la indagación narrativa en la investigación educativa fomenta "la construcción y reconstrucción de las historias personales y sociales; profesores y alumnos son contadores de historias y personajes en sus propias historias y en las de los demás" (Connelly \& Clandinin, 1990:2). Como los seres humanos somos de forma natural narradores de historias, se optó por este método de indagación para explorar las experiencias de los estudiantes utilizando principalmente los datos textuales. Las narraciones se pueden considerar como una acción verbal: "cualquiera que sea la acción particular, cuando alguien cuenta una historia, él o ella le da forma, la construye y la interpreta, experiencia y realidad" (Chase, 2005:657) las narraciones proporcionan no sólo lo que el narrador comunica, sino también su única posición de sujeto. En el análisis se buscaron coherencias y contradicciones entre las historias de los estudiantes, intentando preservar específicamente las palabras únicas e individuales del alumnado ya que reflejan su propio y particular mundo.

\subsection{Sujetos de la investigación}

Los participantes fueron un grupo de catorce estudiantes graduados ese año en la escuela secundaria en una ciudad de tamaño medio en el noreste de Estados Unidos y que se inscribieron como alumnos en el primer año en el Programa Puente de ESL en una universidad estatal. Todos habían mostrado evidencias de motivación académica y de éxito, ya que se habían inscrito en el Programa y habían sido aceptados en él (Fernández y Wassell, 2007).

El propósito del Programa Puente es proporcionar un extenso curso en inglés académico para preparar mejor a los estudiantes con lo que se encontrarán al matricularse en los cursos universitarios regulares. Los alumnos son previamente evaluados al entrar al Programa y colocados de acuerdo a su nivel de competencia en los cursos en los que se realiza un seguimiento. Los niveles van de 1 a 5 , siendo 1 el más básico, el nivel inicial de competencia, y 5 el más alto. Superado éste pueden seguir los cursos regulares de pregrado universitario. 
Todos habían emigrado a los Estados Unidos durante sus años de escuela media o secundaria, y cada uno se había graduado en uno de los dos distritos escolares urbanos cercanos a la universidad. Provenían de ocho países de origen diferentes: Bangladesh (1), Nigeria (1), México (2), República Dominicana (6), Honduras (1), Puerto Rico (1), Camboya (1), y Vietnam (1). Cuatro eran hombres y diez mujeres. Tenían entre 17 y 21 años de edad y estaban en los niveles 4 ó 5 en el momento del estudio, lo que significa que habían demostrado un nivel de aptitud académica en inglés intermedio o superior.

\subsection{Procedimiento de la investigación}

Las autoras asumieron diferentes roles durante el estudio. Además de la dirección de la investigación, la segunda autora también impartió dentro del Programa Puente un curso basado en el Proyecto, con una duración de 5 semanas (40 horas). Aunque las autoras primera y segunda no enseñaron a los estudiantes, se involucraron con el Programa recogiendo datos: la primera autora llevó a cabo todas las entrevistas en español con los estudiantes y estuvo a menudo presente en las clases en calidad de observadora. Como resultado de ello llegó a conocer a muchos de los estudiantes de manera más informal. Las autoras segunda y tercera observaron e interactuaron informalmente con los estudiantes en diferentes contextos, por ejemplo, acompañándoles en las excursiones de sus escuelas secundarias.

\subsubsection{Instrumentos de recogida de datos}

Los datos fueron recogidos durante el Programa Puente en el verano de 2006, y algunos adicionales en el transcurso del cuso académico 2006/2007. Incluyen: transcripciones de las entrevistas (individuales, grupales, e informales); notas de campo basadas en observaciones de las clases puente y otras actividades en las que el grupo participó, incluyendo talleres para maestros; actividades realizadas por ellos (artefactos); y material de archivo de vídeo. Aunque se utilizaron otras fuentes para triangular los resultados, la mayoría de los datos que se presentan provienen de las transcripciones de las entrevistas.

a) Entrevistas: se grabaron 15 horas de entrevistas, en las que cada alumno fue entrevistado individualmente. Su duración oscilaba entre 20 y 30 minutos, desarrollándose en español con los estudiantes de habla hispana y en inglés con los demás. También se realizaron cuatro entrevistas en grupo (en inglés) que se basaron únicamente en quién podía acudir en el momento en que la entrevista había sido programada. Las entrevistas individuales fueron semi-estructuradas y las entrevistas en grupo abiertas, permitiendo así a los estudiantes profundizar en los temas que querían discutir más a fondo. Las entrevistas fueron transcritas, revisadas y analizadas por las tres autoras.

b) Notas de campo y artefactos: se tomaron notas de campo, a veces también grabadas en vídeo, bien como observadores participantes al impartir cursos o como observadores externos en cursos impartidos por otros instructores. Se observó a los estudiantes interactuar de manera informal entre las clases y durante las actividades realizadas dentro del Programa (un picnic de verano en un parque local, un almuerzo de Acción de Gracias celebrado en la cafetería de la Universidad, etc.). Los estudiantes también participaron en un panel de 
expertos durante un seminario organizado para profundizar en el conocimiento del trabajo con los estudiantes ELL en las escuelas. Antes de la sesión, pedimos a los maestros que acudieron que elaboraran preguntas sobre aquellos aspectos de dichos estudiantes por los que sentían curiosidad y que éstos respondieron.

Los artefactos recogidos consistieron principalmente en los trabajos de los estudiantes completados durante las clases del Programa Puente. Debido a la naturaleza abierta del Curso, centrado en una aproximación integradora a la enseñanza de habilidades de lenguaje, se diseñaron actividades de clase en las que los estudiantes fueron capaces de hablar o escribir sobre sus experiencias de vida, incluyendo las que se produjeron durante la escuela secundaria. Todo su trabajo fue recopilado y analizado.

c) Imágenes de vídeo: se grabaron 8 horas de vídeo por los investigadores y por los propios estudiantes. De este modo se recogieron tres excursiones en las que los estudiantes mostraron sus escuelas a los investigadores, comentando sus experiencias en las mismas. Varios estudiantes también llevaron con ellos las cámaras de vídeo a sus casas para documentar sus familias, hogares y comunidades. Los investigadores también grabaron algunas de las actividades de clase que eran pertinentes al estudio.

\subsubsection{Análisis de datos}

Estuvo influido por un enfoque de la teoría de base constructivista (Charmaz, 2005) que hace hincapié en la alineación entre los objetivos de la investigación de la justicia social, la relación entre estructura y actuación, y el análisis de los datos emergentes. De acuerdo a este autor (2003:250), dicha teoría "asume el relativismo de múltiples realidades sociales, reconoce la creación mutua de conocimiento tanto por el observador como por el observado, y tiene como objetivo una comprensión interpretativa de los significados de los sujetos".

La recogida de datos y el análisis de fases fue iterativo y recursivo (Hammersley \& Atkinson, 1983). Los datos se codificaron utilizando el programa HyperResearch de codificación cualitativa. A lo largo de esta fase los investigadores buscaron patrones de coherencia así como contradicciones con dichos patrones (Sewell, 1999; Tobin, 2006). Los datos de vídeo se visualizaron y analizaron utilizando los métodos de análisis de vídeo de Roth (2005). Establecidos los códigos se desarrollaron categorías y temas más amplios (Lichtman, 2006), que incluyeron espacio, tiempo, consideración/preocupación, comprensión hacia los miedos de los estudiantes, y toma de decisiones instruccionales. Se utilizaron notas interpretativas para conectar los códigos y los temas al marco teórico y a otras ideas que surgieron de la literatura (Tobin, 2006). Se utilizaron los criterios de autenticidad de Guba \& Lincoln (1989) para evaluar la calidad de la investigación a lo largo del proceso.

\section{Análisis de los resultados}

Los resultados giran en torno a los principales temas que surgieron de los datos con respecto a la pregunta central a la que trata de responder la presente investigación. Cada uno de estos temas afecta enormemente la actuación de los estudiantes en el aula y por lo tanto tienen fuertes implicaciones para sus profesores. 
Los estudiantes tuvieron muchas oportunidades para hablar en las clases sobre lo que les gusta o disgusta de sus respectivas escuelas secundarias. La mayor parte de sus observaciones describen las estructuras que los maestros crearon mediante sus prácticas: su enseñanza día a día y la gestión del aula. En esencia, los estudiantes indicaron que los maestros que inducían a participar en las prácticas creaban situaciones particulares que o bien facilitaban o bien limitaban académicamente, y a veces socialmente, su éxito.

A continuación se describen los temas que surgieron representando dichas estructuras tanto como recursos como bloqueos relacionados con la capacidad de los estudiantes para actuar. Se ilustra cada tema con las voces de los alumnos aportando nuestra propia interpretación utilizando la visión de la estructura/actuación.

\subsection{Los recursos basados en el aula y en los maestros: espacio, tiempo e interés}

Los datos revelaron que los maestros que potencian y estimulan a los estudiantes ELL para que sean productivos fomentan la existencia de lugares y de momentos para conocer mejor a sus estudiantes, proporcionándoles un acceso adicional al plan de estudios al facilitar oportunidades para que puedan hablar más en inglés. Las formas en que los profesores proporcionaron apoyo, aliento y recursos adicionales a sus estudiantes ELL para favorecer su actuación y éxito como estudiantes consistían en:

a) Apoyo y aliento: para todos los estudiantes ELL en este estudio, el miedo fue un elemento constante en sus experiencias de escolarización; tenían miedo de hablar debido a su limitada habilidad en inglés, y estaban ansiosos porque no entendían la cultura de sus nuevas aulas y escuelas en la que estaban inmersos. Sin embargo, muchos estudiantes describen a maestros concretos que les ayudaron, alentaron, o incluso potenciaron a enfrentarse a estos temores.

Sonia (en entrevista de grupo, mayo de 2006) explicó el miedo que sentía en clase a causa de su incapacidad para comunicarse en inglés. Se sentía más segura escribiendo, aunque reconoce que sólo podía hacerlo "un poco". Sin embargo la profesora proporcionó una importante estructura: su presencia en el aula durante los descansos de diez minutos. Durante este tiempo Sonia podía practicar su inglés hablando y escribiendo. Este tiempo también le permitió a la profesora volver a explicarle el contenido, lo que constituyó un segundo recurso: un acceso adicional al plan de estudios. Por último, la profesora también proporcionó apoyo y refuerzo al tiempo que demostraba su cariño. Reforzó su papel como maestra cuidadora al afirmar que estaba "aquí para enseñar[le]" y reiterando que ella tendría un "buen futuro". Además, recordó a Sonia que aunque la mayoría de sus compañeros, amigos, familia y comunidad hablaban español, para ella era importante que usara inglés para mejorar su fluidez. Volvió a servirle a Sonia como un recurso al mostrarle su tendencia a hablar sólo en su lengua materna y la alentó a que fuera más allá de donde ella se sentía cómoda. Compartiendo sus momentos libres con sus alumnos también mostró su generosidad al prestarles apoyo adicional al aprendizaje.

b) Recursos adicionales: los estudiantes también describieron repetidamente los beneficios al trabajar uno-a-uno con maestros determinados, siendo estos maestros quienes en muchos casos se "extralimitaron" para proporcionar ayuda adicional y estímulo. Alicia (en 
entrevista individual, diciembre de 2006) describió la importancia del espacio y el tiempo extra que una profesora le proporcionó al percatarse de que podría necesitar apoyo cuando Alicia realizó mal un examen. Le suministró libros y tareas adicionales, y también le hizo hablar en inglés para que tuviera mayor destreza fuera del aula. Ambas prácticas por parte de la profesora ayudaron a facilitar el éxito de Alicia, no sólo en clase, sino también a ser más competente hablando inglés.

La mayoría de los estudiantes mencionó que sólo algunos profesores les proporcionaron estos recursos. Por ejemplo, Marta dijo: "Entre muchos maestros, encuentras a uno que se preocupa y quiere que aprendas" (en entrevista de grupo, mayo de 2006). Por su parte, Zenaida (en entrevista individual, mayo de 2006) valoró que su profesor se interesara por conocer algo sobre ella y sobre su cultura. Era importante para ella tener un maestro en quien pudiera confiar. Describe a profesores que manifestaron no sólo un respeto por ella como estudiante, sino también un nivel de atención que le facilitó su éxito. Esta confianza y respeto la llevó a sentirse cómoda hablando inglés, lo que le proporcionó un ambiente seguro para ser más experta, donde también podía compartir los problemas que estaba teniendo en la escuela o en su adaptación a la vida en los Estados Unidos.

El grupo de estudiantes reiteró cómo los profesores deberían "ser amigables con los estudiantes" (de la transcripción de trabajos de los estudiantes, agosto de 2006). Para ellos es importante tener un adulto con quien poder conectar y confiar preguntas sobre la escuela, el lenguaje, la comunidad, etc. ya que deben enfrentarse a nuevas culturas en múltiples niveles.

\subsection{El maestro y los obstáculos en el aula}

Los datos mostraron que los profesores también se unen a prácticas que pueden crear estructuras que actúan como barreras para el éxito y el aprendizaje de los estudiantes ELL. En algunos casos, éstas tuvieron efecto en el nivel de comodidad de los estudiantes en las clases, lo que repercutió en el aprendizaje del contenido y en las oportunidades para ser más competentes en inglés. Estos obstáculos incluyen casos en los que los maestros impidieron su acceso al plan de estudios, utilizaron malas prácticas de instrucción, escasa comprensión de su miedo a comunicarse en inglés, o perpetuaron ideas preconcebidas sobre los estudiantes.

a) Acceso al plan de estudios: algunos profesores diluían el plan de estudios, proporcionando una mínima instrucción, o utilizando materiales que simplemente no eran suficientemente desafiantes para los estudiantes ELL. En una de las entrevistas en grupo pedimos a los estudiantes que describieran las cosas que cambiarían de los maestros que tuvieron en la escuela secundaria. Abul, un estudiante de Bangladesh, describió su experiencia: "Creo que algunos maestros iban al aula sólo a dormir, sólo encendían el televisor y veíamos cualquier película o algo así. Tal vez, yo cambiaría eso" (en entrevista de grupo, mayo de 2006). Catalina también describe clases donde se llevó a cabo una instrucción poco estimulante: "Pasé como tres semanas con el mismo tema, los estudiantes sólo iban a clase para jugar, ver la televisión, bailar, lo que sea y no aprendí nada en esa clase" (en entrevista individual, julio de 2006).

Para Catalina, Abul y otros, repetir los contenidos del plan de estudios o ni tan siquiera recibirlos no sólo no les ayudó académicamente sino que por el contrario les hizo perder 
contenidos adicionales que hubiesen ampliado claramente sus conocimientos. Además, con independencia de que televisión, películas, juegos y bailes se llevaran a cabo una vez al año o una vez a la semana, hay que señalar que estos profesores perdieron un valioso tiempo de instrucción. Si los estudiantes ELL deben mejorar su dominio del inglés y alcanzar los mismos estándares que sus compañeros de habla inglesa, no es aceptable que tengan excesivo tiempo libre o entretenimiento durante las clases.

b) Decisiones de instrucción: en algunos casos, este acceso al plan de estudios se vio dificultado aún más por decisiones de los profesores sobre la instrucción, en concreto obligándoles a hablar en el aula sólo en inglés, no permitiéndoles discutir o comentar con sus compañeros, e implementando de manera muy pobre actividades de aprendizaje cooperativo, precisamente cuando el fomento de estrategias sociales como la cooperación y la interacción entre los alumnos para organizar, evaluar y practicar el desarrollo en el uso de las distintas destrezas son destacadas por algunos autores (Franco, 2004), como necesarias a la hora de diseñar y aplicar modelos de enseñanza estratégica.

Cuando se les preguntó qué no deben hacer los maestros cuando se trabaja con los estudiantes ELL, los participantes reiteraron que "no deberian hacer que los alumnos sólo hablen en el aula en inglés" (del artefacto de trabajo de los estudiantes, julio de 2006). Si bien métodos seguros de enseñanza aprueban el uso de la primera lengua de los estudiantes para aclarar dudas sobre el contenido (Echevarria, Vogt \& Short, 2011), los participantes en nuestro estudio habían estado en aulas en las que no se les permitía hablar su lengua materna. Ajani, un estudiante de Nigeria, también describió un aula en la que hubo muy poca interacción, excepto entre el estudiante y el profesor: "Pero una cosa que no me gusta es no nos deja hablar con el compañero. Cuando tenemos una pregunta sólo podemos preguntarle [al profesor]. Eso no me gusta" (en entrevista de grupo, mayo de 2006). Los estudiantes ELL necesitan oportunidades en las que puedan hablar con sus compañeros para aclarar el significado, pues los mismos estudiantes pueden ser un poderoso recurso a la hora de ayudar en el aprendizaje a sus compañeros. Cuando los profesores no permiten a los estudiantes hablar en su lengua materna o preguntar a otros estudiantes les están limitando su acceso a un importante recurso basado en el aula, lo que afecta a su actuación como aprendices.

Abul describió una situación problemática que se daba a menudo cuando los profesores iniciaban una actividad de aprendizaje cooperativo: "Y otra cosa, en la clase, al trabajar en grupo, ¿sabes cómo escoger pareja en clase? Te dicen que escojas pareja y todo el mundo va con sus propios compañeros para trabajar juntos en grupo y entonces sólo quedo yo y tengo que hacer el trabajo solo" (de la transcripción de un taller, noviembre de 2006).

Durante la sesión de trabajo en la que Abul describió este escenario, otros estudiantes asintieron con la cabeza pues ellos también habían sufrido algo similar (notas de campo de un taller, noviembre de 2006). Desafortunadamente, permitir que los alumnos elijan a sus parejas es una práctica que muchos profesores utilizan a menudo. No obstante, esta estrategia inmediatamente desalentó a Abul. El hecho de que tuviera que trabajar solo contradijo el punto de la lección: que los estudiantes trabajaran juntos colaborando para compartir perspectivas y aprender unos de otros. No se le permitió acceder al recurso de sus compañeros, lo que cambió para él la actividad de aprendizaje. Abul también perdió un tiempo valioso en el que podía haber interactuado con sus compañeros en inglés, una práctica instruccional que para 
los alumnos ELL es esencial para mejorar su destreza. Ser rechazado por sus compañeros también impactó el sentido de conexión de Abul con los otros estudiantes en la clase. Como resultado final, la actuación de Abul se vio afectada por su incapacidad para acceder a sus compañeros como un recurso y por sus sensaciones de rechazo por la clase.

c) Miedo y menosprecio: algunos profesores tenían escasa empatía hacia (o incluso desconocían) el miedo de los estudiantes ELL a comunicarse en inglés y a integrarse en una nueva cultura. Los comentarios de Eduardo (de la transcripción de un taller, octubre de 2006), al explicar la multitud de miedos que sentía como recién llegado a su escuela secundaria, enfatizaron un punto fuerte que de forma consistente surgía de los datos. Él y muchos otros estudiantes experimentaron miedo a dos niveles: al hablar inglés y al tratar con la cultura de la escuela, la comunidad y los Estados Unidos en general. Dudaba al hablar en inglés en clase porque pensaba que su acento era pobre. Curiosamente, en su clase había muchos estudiantes puertorriqueños que eran hispanohablantes nativos, pero, como un inmigrante dominicano recién llegado, Eduardo no sentía ninguna conexión con ellos porque tenían un alto nivel de destreza en inglés. A su vez, tenía miedo de hablarles. También indicó que no había nadie en su familia que pudiera ayudarle a afrontar las nuevas culturas que experimentaba. Como muchos otros recién llegados, sus padres trabajaban muchas horas y tenían poco tiempo para apoyarle en su adaptación a la escuela y en su aprendizaje del inglés.

Eduardo también describió una comunidad en clase en la que tenía mucho miedo de asumir riesgos para comunicarse y así aprender mejor el idioma. A pesar de que él no describió ejercicios concretos en los que el profesorado le involucrara, el esquema o ideología seguido en la comunidad de la clase hacía que se sintiera incómodo y perpetuara su miedo a hablar en inglés.

Sarita, otra estudiante que habló en el panel de expertos, describió situaciones en las que los profesores no intervenían cuando otros estudiantes se reían de los intentos de los estudiantes ELL por usar inglés (de la transcripción de un taller, octubre 2006). Ella reiteró el temor que sentía cuando sus compañeros criticaban sus habilidades lingüísticas en el aula. Indicó también el intenso deseo que tenía de regresar a su país de origen, donde podía comunicarse sin hacer frente a quienes la hacían sentir "estúpida". Aunque los estudiantes pueden menospreciar a los otros fuera del aula, se desprende de los datos que el profesorado tiene que asumir la responsabilidad de crear en el aula un ambiente donde el alumnado se sienta seguro y cómodo, especialmente si se espera que adquiera el dominio del lenguaje. A pesar de que los aprendices suelen considerar como demeritorio hablar con "acento" extranjero porque creen que revela su incapacidad para acceder a la lengua "normal" (Moreno, 2007), no es de extrañar que tengan una tendencia a aferrarse a su lengua materna y no se atrevan a comunicarse en inglés si se encuentran en el aula con situaciones donde son menospreciados e incluso ridiculizados.

En los escenarios descritos por los participantes los maestros crearon estructuras en las aulas que desalentaron a sus estudiantes ELL. Los profesores implicados quizá no fueron conscientes de la burla, la risa o la crítica que tuvieron lugar. Sin embargo, los estudiantes angloparlantes en el aula mantuvieron el esquema, o las creencias implícitas, de que era aceptable hacer comentarios y reírse de los estudiantes con acentos. Este esquema dio forma a la actuación de los estudiantes ELL ya que les dificultó hablar en clase. Por no hacerlo, tenían menos oportunidades de preguntar, participar en actividades cooperativas de aprendi- 
zaje, o discutir ideas con los compañeros o con el profesor. Al no participar en estos actos de comunicación se vieron afectadas su actuación como estudiantes, su comprensión del contenido y su capacidad para practicar inglés.

d) Ideas preconcebidas en el aula: los estudiantes también describieron algunas de las formas en las que se sentían incomprendidos en el aula, lo que ocurría cuando ellos evitaban hablar por temor a no ser entendidos, o cuando los maestros confundían su timidez con una falta de interés por hablar inglés.

Verónica (en entrevista individual, noviembre de 2006) creía que ni sus maestros ni sus compañeros la entendían, por lo que rara vez hablaba en clase. Se sintió desalentada, y como resultado no fue un miembro activo de la comunidad educativa, a pesar de que sus profesores continuamente la animaban a participar: "Ellos [los profesores] siempre me dicen que yo tengo que participar más, pero... no sé, yo no puedo ser así (sonrie avergonzada). A veces es que ellos no me comprenden, no entienden".

Otra estudiante, María (en entrevista individual, enero de 2007), también relató un malentendido común que se produjo entre ella y algunos de sus maestros. Se describió a sí misma como muy tranquila y tímida en clase. Sus maestros interpretaron su tranquilidad y su silencio como una actitud despreocupada sobre la clase. Curiosamente, María señaló que no era necesariamente su miedo a hablar inglés, sino simplemente su personalidad tranquila: "Entonces yo pienso que [los maestros] debían de... saber de que no todos los estudiantes somos iguales, algunos somos asi más callados pero sí nos importa la clase pero no nos gusta hablar. Y eso es lo que pasa conmigo en las clases, por eso creo que tengo problemas".

Es frecuente entre el profesorado dejarse llevar por el efecto Halo: cuando un maestro desarrolla un esquema o creencias sobre la actitud de un estudiante, el esquema puede afectar a sus interacciones con el estudiante. Juicios como los realizados por los profesores de María pueden afectar al trabajo y a los logros de los estudiantes.

\section{Conclusiones}

Los hallazgos muestran que el profesorado participa en las prácticas específicas y crea estructuras en las aulas que tanto potencian como restringen el aprendizaje de los estudiantes ELL y el nivel de comodidad en la clase. En términos de estructuras, ciertos recursos, como espacio, tiempo, y afecto o atención, facilitaron la actuación del alumnado. El elemento espacio incluyó situaciones uno-a-uno con los maestros en las que los estudiantes podían practicar inglés, recibir apoyo y acceder a contenidos complementarios. También incluyó encuentros con otros estudiantes para construir relaciones y aprender unos de otros. Cuando los profesores disponían de tiempo adicional para los estudiantes, ya sea formal o informalmente, ofrecieron un apoyo suplementario para el aprendizaje y una motivación personal. El espacio se convirtió en un recurso físico apoyado por el esquema o sistema de creencias en el que el tiempo y la disponibilidad por parte del profesor era una prioridad para los estudiantes ELL; sabían qué profesores les ayudarían a través de sus tutorías o de sesiones de discusión uno-a-uno que podían tener lugar después de la escuela, durante el almuerzo o entre las clases. Por último, ambos elementos de espacio y tiempo quedaron 
fuertemente conectados por una disposición generosa del profesorado hacia sus alumnos. Los estudiantes ELL valoraron a los maestros que se preocuparon por apoyarles y alentarles ya que se enfrentaban a algunos desafíos descritos por otros investigadores, como la obtención de competencia en inglés mientras aprenden los contenidos (Short \& Fitzsimmons, 2007) y hacer frente a la exclusión y a otros problemas sociales en el contexto escolar (Serra i Salame, 2004; Fernández y Wassell, 2007). Estos recursos particulares influyeron claramente en la actuación de los estudiantes, ya que facilitaron el aprendizaje, el uso adicional del inglés, y un mayor sentido de aceptación y comodidad en las aulas.

Por otro lado, los resultados indican que las estructuras específicas también impidieron la actuación de los estudiantes ELL. La mayoría de estos obstáculos derivan de prácticas de enseñanza que disminuyen el pleno acceso al plan de estudios, tales como diluir dicho plan, repetir lecciones, reemplazar clases con la televisión o cualquier otro entretenimiento, limitar el acceso de los estudiantes a la ayuda de los compañeros, o políticas en el aula de sólo inglés. Estos resultados apoyan las conclusiones alcanzadas por Gándara et al. (2003), que afirman que los estudiantes ELL tienen acceso a un plan de estudios generalmente inferior y cubierto en un período de tiempo menor de lo habitual. Cuando los estudiantes ELL experimentan estas prácticas de enseñanza, las desigualdades son inevitables, ya que a su vez tienen menos oportunidades de acceso a los contenidos que necesitan para superar las pruebas de alta participación, de prepararse para la universidad o el mundo laboral, y para aprender inglés, lo que afecta claramente a su actuación o a su capacidad para acceder a los recursos como aprendices.

Tales hallazgos sugieren un concepto fundamentalmente diferente para las mejores prácticas del maestro con los estudiantes ELL, que no son necesariamente de naturaleza pedagógica. Por el contrario, en su mayor parte se basan en la atención y empatía hacia los estudiantes y sus situaciones particulares como estudiantes de inglés y de contenidos. Además, los maestros deben entender que los estudiantes ELL están negociando lo que significa ser estudiantes de secundaria, miembros de nuevas comunidades y nuevos ciudadanos estadounidenses. Si su éxito escolar está en parte regido por influencias contextuales de la escuela y ajustes de la personalidad (Portes \& Rumbaut, 2001), debemos resaltar la importancia de ofrecer tiempo, espacio y aliento. También debemos reconocer el efecto perjudicial de prácticas de enseñanza insuficientes, el acceso reducido al plan de estudios, y los casos en los que los temores de los estudiantes, sus retos, sus preocupaciones y sus identidades únicas no son tenidos en cuenta.

De este estudio se pueden extraer varias implicaciones fuertes para el profesorado. Los resultados sugieren que los profesores necesitan desarrollar un profundo conocimiento y sentido de empatía por la situación única de sus estudiantes como aprendices y como individuos. Los estudiantes ELL necesitan sentirse parte de una comunidad y saber que sus profesores se preocupan por ellos. Este sentido de empatía y comprensión ha de servir como base pedagógica para la toma de decisiones relacionadas con la enseñanza. Para llevarlo a cabo los maestros tienen que emplear tiempo para hablar con ellos y obtener una clara visión de sus necesidades, desafíos y expectativas. Dentro de este marco, los profesores deben ofrecer a los estudiantes ELL oportunidades tanto en clase como fuera de ella para hablar y para proporcionarles ayuda adicional cuando sea necesario.

¿Cómo pueden los maestros sacar tiempo para conocer a sus alumnos y comenzar a desarrollar una mayor comprensión sobre sus vidas y sus situaciones? En esta investigación 
invitamos a grupos de estudiantes ELL del Programa Puente universitario para servir como panel de expertos en talleres para maestros de primaria y secundaria $(\mathrm{K}-12)$ centrados en satisfacer las necesidades de ELL en las clases regulares. Estas sesiones resultaron ser el elemento más poderoso de los talleres, ya que los estudiantes acababan de graduarse en la escuela secundaria y a menudo estaban dispuestos a compartir sus historias -tanto buenas como malas- con los maestros en ejercicio.

\section{REFERENCIAS BIBLIOGRÁFICAS}

Aud, S., Hussar, W., Johnson, F., Kena, G., Roth, E., Manning, E., Wang, X. and Zhang, J. (2012). The Condition of Education 2012 (NCES 2012-045). Washington, DC: U.S. Department of Education, National Center for Education Statistics. Available from: http://nces.ed.gov/ pubsearch, accessed 1 August, 2013.

Batalova, J. and McHugh, M. (2010). Number and Growth of Students in US Schools in Need of English Instruction. Washington, DC: Migration Policy Institute. Available from: http:// www.migrationinformation.org/ellinfo/FactSheet_ELL1.pdf, accessed 1 August, 2013.

Charmaz, K. (2003). "Grounded theory: Objectivist and constructivist methods", in N.K. Denzin e Y.S. Lincoln (eds.), Strategies of qualitative inquiry ( $2^{\mathrm{a}}$ ed.). London: Sage Publications Limited, 249-291.

Charmaz, K. (2005). "Grounded theory in the 21st Century: Applications for advancing social justice studies", in N.K. Denzin e Y.S. Lincoln (eds.), Handbook of qualitative research ( $3^{\mathrm{a}}$ ed.). Thousand Oaks, CA: Sage, 507-536.

Chase, S.E. (2005). "Narrative inquiry: Multiple lenses, approaches, voices", in N.K. Denzin e Y.S. Lincoln (eds.), The Sage handbook of qualitative research ( $3^{\mathrm{a}}$ ed.). Thousand Oaks, CA: Sage, 651-679.

Connelly, F.M. and Clandinin, D.J. (1990). "Stories of experience and narrative inquiry", en Educational Researcher, 19: 2-14.

Cosentino de Cohen, C., Deterding, N. and Chu Clewell, B. (2005). Who's left behind?: Immigrant children in high and low LEP schools. Program for Evaluation and Equity Research, Washington, DC: Urban Institute.

Echevarria, J., Vogt, M. and Short, D. (2011). Making content comprehensible for English Learners: The SIOP Model (4a ed.). Boston, MA: Pearson Allyn \& Bacon.

Fernández Hawrylak, M. and Wassell, B.A. (2007). Experiencias de los estudiantes inmigrantes en High Schools urbanos. En J. Ipland García et al. (eds.), Actas en CD-ROM del IV Congreso Internacional y XXIV Jornadas de Universidades y Educación Especial "Atención a la diversidad, una responsabilidad compartida". Huelva: Universidad de Huelva.

Franco Naranjo, P. (2004). "El uso de estrategias de aprendizaje del inglés como lengua no materna: aplicación de cuestionario como fase previa a la enseñanza de estrategias en el aula". Porta Linguarum 2: 57-67, available from: http://www.ugr.es/local/portalin, accessed 8 November, 2010.

Gándara, P. (2010). "The Latino Education Crisis", in Educational Leadership, 67, 5: 24-30.

Gándara, P., Rumberger, R., Maxwell-Jolly, J. and Callahan, R., (2003). "English Learners in California Schools: Unequal resources, unequal outcomes". Education Policy Analysis Archives 11, 36: 1-54, available from: http://epaa.asu.edu/epaa/v11n36/, accessed 8 July, 2007.

González, J. and Darling-Hammond, L. (1997). New concepts for new challenges: Professional development for teachers of immigrant youth. Washington, DC: Center for Applied Linguistics. 
Guba, E.G. and Lincoln, Y.S. (1989). Fourth generation evaluation. Newbury Park, CA: Sage.

Gutiérrez, K. and Orellana, M. (2006). "The problem of English Learners: Constructing genres of difference", in Research in the Teaching of English, 40: 502-507.

Hakuta, K., Butler, Y.G. and Witt, D. (2000). How long does it take English Learners to attain proficiency. The University of California Linguistic Minority Research Institute. Policy Report 2000-1. Available from: http://escholarship.org/uc/item/13w7m06g, accessed 8 July, 2007.

Hammersley, M., and Atkinson, P. (1983). Ethnography: Principles in practice. Londres: Routledge.

Lichtman, M. (2006). Qualitative research in education: A user's guide. Thousand Oaks, CA: Sage.

Louie, V. (2005). "Immigrant newcomer populations, ESEA, and the pipeline to college: Current considerations and future lines of inquiry", in Review of Research in Education, 29: 69105.

Moreno Fernández, F. (2007). “Adquisición de segundas lenguas y Sociolingüística”, in Revista de Educación, 343: 55-70.

Noguera, P.A. (2003). City schools and the American Dream. New York, NY: Teachers College Press.

Orellana, M. and Gutiérrez, K. (2006). "What's the problem? Constructing different genres for the study of English Learners", in Research in the Teaching of English, 41: 118-123.

Portes, A. y Rumbaut, R.G. (2001). Legacies: The story of the immigrant second generation. Berkeley, CA: University of California Press.

Roth, W.M. (2005). Doing qualitative research: Praxis of method. Rotterdam: Sense Publishers.

Sacks, A. and Watnick, B. (2006). "A snapshot of teacher perceptions on full inclusion in an international urban community: Miami-Dade County, Florida", in Journal of the International Association of Special Education, 7: 67-74.

Serra i Salame, C. (2004). "Rhetoric of exclusion and racist violence in a Catalan secondary school", in Anthropology \& Education Quarterly, 35: 433-450.

Sewell, W. (1992). "A theory of structure: Duality, agency, and transformation", in American Journal of Sociology, 98: 1-29.

Sewell, W.H. (1999). "The Concept(s) of Culture", in V. Bonnell y L.E. Hunt (eds.), Beyond the Cultural Turn. Berkeley, CA: University of California Press, 35-61.

Short, D. and Fitzsimmons, S. (2007). Double the work: Challenges and solutions to acquiring language and academic literacy for adolescent English language learners - A report to Carnegie Corporation of New York. Washington, DC: Alliance for Excellent Education.

Tobin, K. (2006). "Qualitative research in classrooms: Pushing the boundaries of theory and methodology", in K. Tobin y J. Kincheloe, (eds.), Doing educational research - A handbook. Rotterdam: Sense Publishers, 15-58.

Vang, C.T. (2005). "Minority students are far from academic success and still at-risk in public schools", in Multicultural Education, 12: 9-15.

Wassell, B.A.; Hawrylak, M.F. and LaVan, S.K. (2010). "Examining the structures that impact English language learners' agency in urban high schools: Resources and roadblocks in the classroom", in Education and Urban Society, 42, 5: 599-619. 